\title{
[Letter to the Editor]
}

\section{Protein phosphorylation status is preserved following dual RNA and protein extraction using the Qiagen RNeasy Mini Kit} and protein maximize the biological information obtained from cell or tissue samples while avoiding the need for large sample numbers. Consequently, significant advances have been made in commonly used methodologies, such as the column-based RNeasy Mini Kit (Qiagen, Mississauga, ON, Canada), that permit researchers to study the expression level of a specific gene and its corresponding protein from the same sample (1). These techniques allow for the elution of total RNA with sufficient quality and quantity for microarray analysis and cDNA generation, as well as adequate yields of protein for immunoblots. Moreover, columnbased kits do not require hazardous chemicals, such as phenol or chloroform, or time-consuming cesium chloride $(\mathrm{CsCl})$ gradient centrifugation (1). However, it is not known if protein phosphorylation status is conserved
Methods for dual extraction of total RNA

when extracting proteins using columnbased kits since this approach does not use protease inhibitors. Here, we show that the phosphorylation status of both protein kinase B/Akt, an important signaling protein that mediates cell growth, survival, proliferation, and insulin-stimulated glucose uptake, and the transcription factor Stat3 is preserved in the flow-through fractions of cell and tissue samples processed with the RNeasy Mini Kit compared with a classical protein isolation methodology.

The RNeasy Mini Kit and the techniques outlined in the associated handbook (www.qiagen.com/ca/ resources/resourcedetail $\mathrm{id}=14 \mathrm{e} 7 \mathrm{cf} 6 \mathrm{e}$ 521a-4cf7-8cbc-bf9f6fa33e24\&lang=en) were used to simultaneously harvest total RNA and protein from samples of rat skeletal muscle L6 myotubes ( 200,000 cells) (ATCC CRL-1458; ATCC, Manassas, VA), mouse 3T3-L1

A

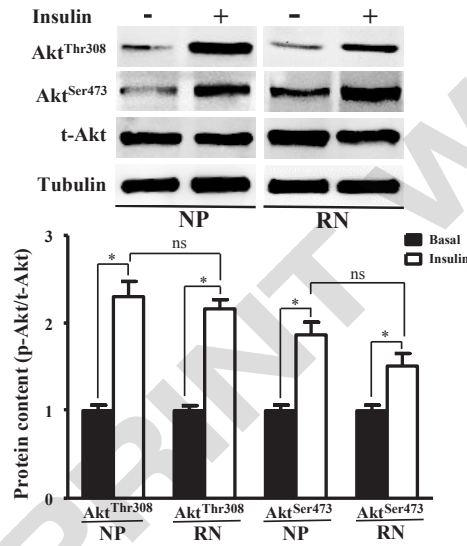

C

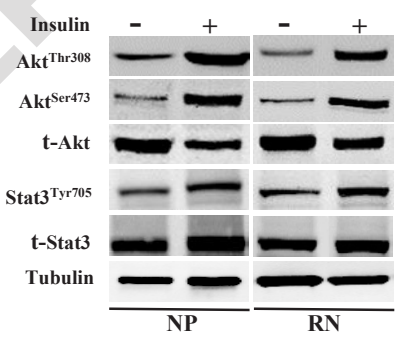

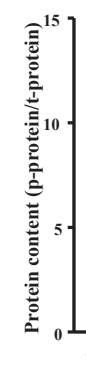

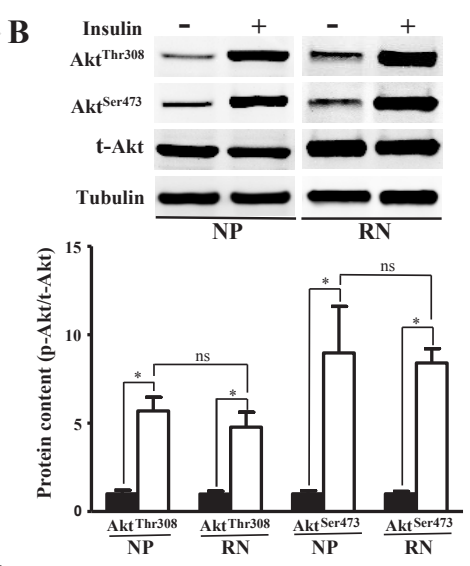

adipocytes ( 135,000 cells) (ATCC CRL-173; ATCC), and Sprague Dawley rat visceral epididymal white adipose tissue (EWAT) ( $30 \mathrm{mg}$ ). Cultured cells were scraped, lysed in RLT buffer, and collected using cell scrapers (Fisher Scientific, Ottawa, ON, Canada). EWAT samples were homogenized in RLT buffer using pellet pestles (Sigma, Oakville, ON, Canada) and passed through a $27 \mathrm{G} \times 1 / 2$ inch needle. To remove lipid and tissue debris, EWAT homogenates were centrifuged at 1500 $\times g$ for $5 \mathrm{~min}$, and the aqueous phase was collected. Following the RNeasy Mini Kit instructions, 70\% ethanol was added to cell lysates or EWAT homogenates, and the samples were mixed by pipetting prior to being transferred to columns. After centrifugation, the flow-through extract was retained and stored on ice. Isolation of total RNA was performed as described in the RNeasy Mini Handbook without modifi-

Figure 1. Comparative immunoblot analysis of L6 myotube, 3T3-L1 adipocyte and epididymal white adipose tissue (EWAT) lysates using the classical NP40 lysis buffer method and the RNeasy Mini Kit. Comparing the two protein extraction methods revealed that $A k^{T h r 308}$ and $A k t^{S e r 473}$ immunoblots show equivalent responses to insulin treatment in $L 6$ cells (A), 3T3-L1 cells (B) and EWAT (C). Changes in Stat3 ${ }^{\text {Tyr705 }}$ in EWAT (C) after insulin stimulation were also similar when comparing the two protein extraction methods. Representative immunoblots and protein fold-changes are displayed. Data correspond to mean \pm SEM. NP: NP40 lysis buffer; RN: RNeasy Mini Kit. 3T3-L1 adipocytes were treated with $100 \mathrm{nM}$ insulin/well (+) or no insulin (-) for $10 \mathrm{~min}$. Differentiated L6 cells were treated with $100 \mu \mathrm{U} / \mathrm{mL}$ insulin or no insulin for $10 \mathrm{~min}$. EWAT was collected from male rats at basal state (-) and following $10 \mathrm{~min}$ injection of $10 \mathrm{U}$ insulin/kg body weight. * indicates $P<0.05$ when comparing changes in phosphorylation status between basal and insulin-stimulated conditions within each extraction method; ns: not significant when comparing phosphorylation status in insulin-stimulated conditions between the two extraction methods. 


\section{FROM THE INVENTORS OF THE}

..............

197mas:

$\bullet \bullet \bullet \bullet \bullet \bullet \bullet \bullet \bullet \bullet \bullet \bullet \bullet \bullet \bullet-1: 1 \quad 1$ in

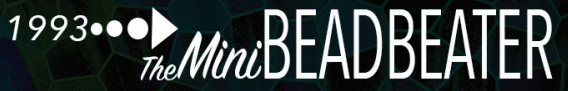
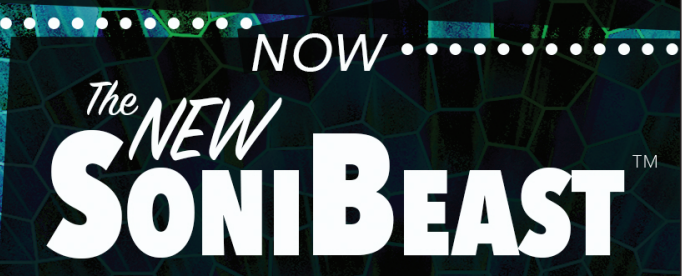

$\bullet \bullet \bullet \bullet \bullet \bullet \bullet \bullet \bullet \bullet \bullet \bullet \bullet \bullet \bullet \bullet$

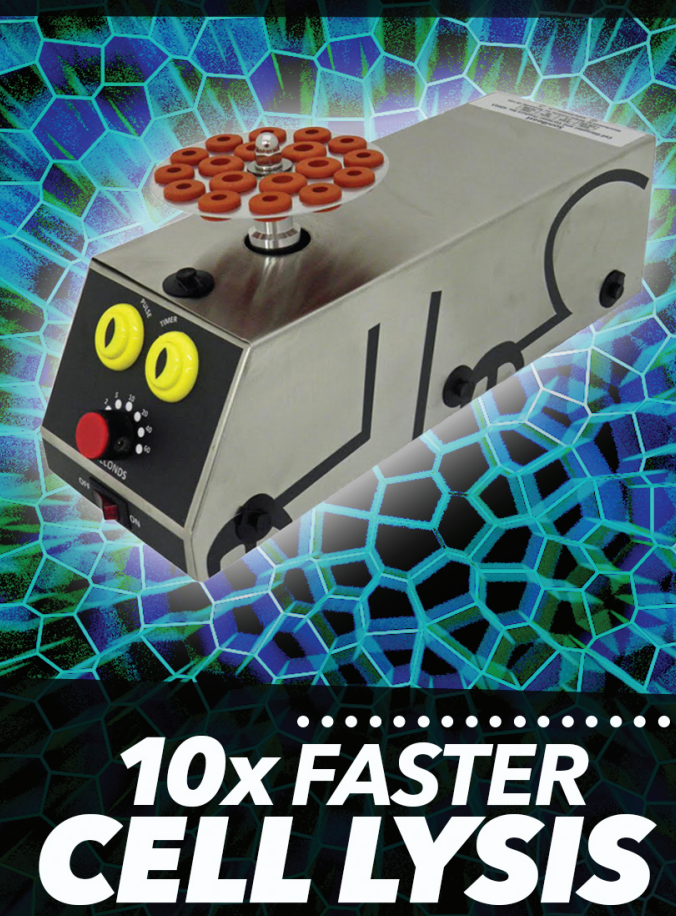

T.

- SMALL SAMPLE SIZE

- SIMPLE OPERATION

- LARGE CAPACITY

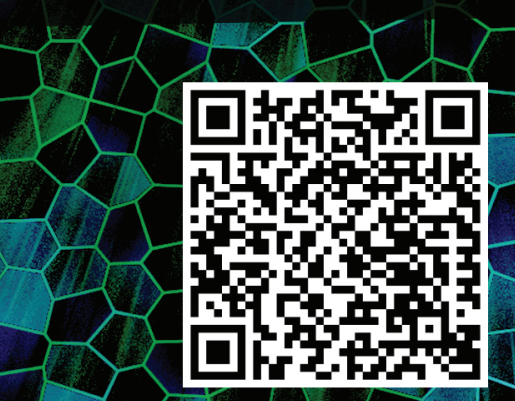

BIOSPEC PRODUCTS WWW.BIOSPEC.COM

L6 samples
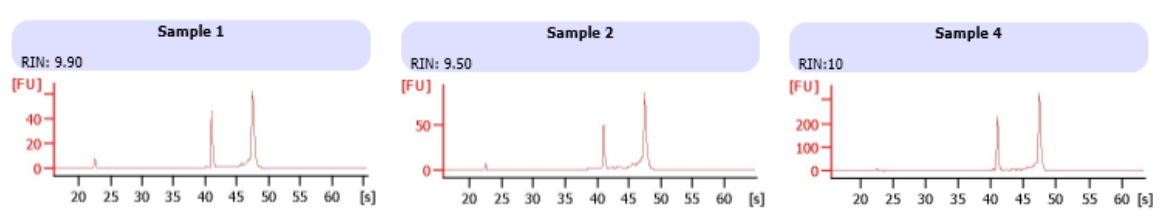

3T3-L1 samples
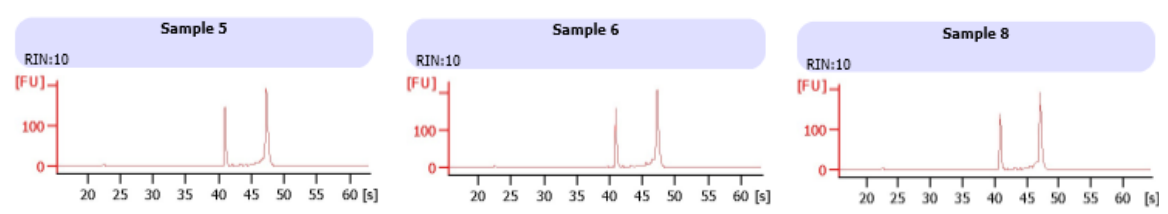

EWAT samples
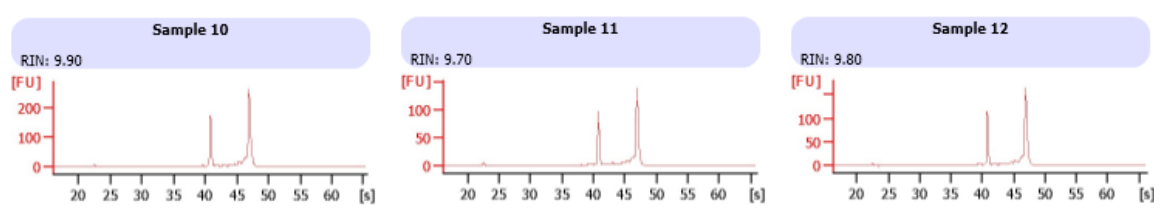

Figure 2. Agilent 2100 Bioanalyzer analysis of RNA collected from L6 myotubes, 3T3-L1 adipocytes and epididymal white adipose tissue (EWAT). Total RNA was run on an Agilent 2100 Bioanalyzer using RNA Nano Chips to determine sample quality. Representative electropherograms showing $18 \mathrm{~S}$ and $28 \mathrm{~S}$ ribosomal peaks are provided for three L6 cell samples, three 3T3-L1 cell samples, and three EWAT samples. The RNA ribosomal integrity numbers (RIN) of the samples ranged from 8.9 to 10 , indicating high-quality RNA

cation. RNA quality was assessed with an Agilent 2100 Bioanalyzer (Agilent Technologies, Waldbronn, Germany). Ice-cold acetone (4 v/v) was added to the flow-through extracts, and samples were left overnight at $-20^{\circ} \mathrm{C}$, leading to the formation of a white protein precipitate. The flow-through/acetone/precipitate mix was then centrifuged at 15,700 $\times g$ for $10 \mathrm{~min}$ to remove acetone and any traces of salts contained in the various RNeasy Mini Kit buffers. The supernatant was discarded, leaving an isolated pellet. Protein pellets were air-dried for $10 \mathrm{~min}$ and then resuspended in $400 \mu \mathrm{L} 10 \%$ sodium dodecyl sulfate (SDS).

For this study, proteins collected with the RNeasy extraction method were compared with those collected with the classically used NP40 protein extraction method (2). The classical method includes lysing cells or homogenizing tissues in NP40 lysis buffer (Fisher Scientific) using cell scrapers and pellet pestles, respectively. In contrast to the RNeasy extraction method, the NP40 lysis buffer contains a cocktail of protease inhibitors (Sigma) and phenylmethylsulfonyl fluoride phosphatase inhibitors (Sigma). For the current analysis, EWAT samples were centrifuged at $1500 \times g$ at $4^{\circ} \mathrm{C}$ for $5 \mathrm{~min}$ to remove lipid and tissue debris, while cell lysates were vigorously vortexed prior to protein analysis.

The protein concentration of the lysates was determined using the DC Protein Assay (Bio-Rad, Mississauga, ON, Canada). Protein expression was analyzed by separating $30 \mu \mathrm{g}$ of protein extracts on 10\% SDS-polyacrylamide electrophoresis gels. Proteins were then transferred to polyvinylidene difluoride membranes (Millipore, Billerica, MA). Equal loading of protein was verified by immunodetection of $\alpha$-tubulin (Abcam, Cambridge, MA). The following antibodies were used: Akt (\#07-372; Millipore), Akt ${ }^{\text {Ser473, }}$ Akt $^{\text {Thr308 }}$, Stat3 ${ }^{\text {Tyr705 }}$, Stat3 (\#9271S, \#9275S, \#9131, \#79D7, respectively; Cell Signaling Technology, Whitby, ON, Canada), and anti-rabbit IgG (711-035152; Jackson ImmunoResearch Laboratories, Jennersville, PA). Blots were visualized with an enhanced chemiluminescence western blotting detection kit (Western Lighting Plus-ECL, PerkinElmer, MA) and analyzed using the 
AlphaView software (Cell Biosciences, Santa Clara, CA).

As shown in Figure 1 and Supplementary Figure 1, immunoblots of common phosphorylation sites on Akt $\left(\mathrm{Thr}^{308}\right.$ and Ser ${ }^{473}$ ) and Stat3 $\left(\mathrm{Tyr}^{705}\right.$ ) show equivalent responses to insulin with both protein extraction methods. Average RNA yields of 15.0, 9.0, and $4.5 \mu \mathrm{g}$ were obtained from L6 cells, 3T3-L1 cells, and EWAT, respectively. As shown in Figure 2, RNA samples collected from the RNeasy Mini Kit all showed ribosomal integrity numbers (RIN) between 8.9 and 10, confirming that this modified method for protein extraction does not compromise RNA quality.

A previous study (1) using the RNeasy Mini Kit demonstrated concurrent RNA and protein extraction from the same sample; however, the authors focused on non-phosphorylated proteins. While modified TRIzol-based methods (3) can be used to analyze the phosphorylation status of proteins recovered from samples simultaneously processed for nucleic acid extraction, these methods require the use of hazardous organic solvents such as phenol and chloroform in addition to TRIzol. Our data confirm the preservation of phosphorylation status of proteins in flow-through fractions collected using the columnbased RNeasy Mini Kit. This is of substantial value, since the proteincontaining fraction is typically discarded by researchers. Here, we have demonstrated that RNeasy column-based protein extraction does not compromise the phosphorylation status of phosphoproteins, which is particularly important given that this post-translational modification is a key mechanism for regulating protein function and activity (4). Moreover, it is well-known that many metabolic processes, such as signal transduction, transcriptional regulation, and cell division, are regulated via protein phosphorylation (5). As such, the use of dual RNA and protein extraction by column offers significant advantages for researchers, including reducing experimental costs and sampling errors, allowing the use of smaller sample numbers in research studies, and maximizing the information obtained from biological samples when a limited amount of material is available.

\section{Author contributions}

O.S. and D.M.M. conceived the idea for the study, analyzed the data, and wrote the manuscript. O.S. and D.J.D. performed the experiments.

\section{Acknowledgments}

The authors thank Steve Dragos for his assistance with 3T3-L1 cell culture, Daniel Cervone for animal husbandry, and Katherine Suitor for RNA integrity assessment.

\section{Competing interests}

The authors declare no competing interests.

\section{References}

Morse, S.M., G. Shaw, and S.F. Larner. 2006 Concurrent mRNA and protein extraction from the same experimental sample using a commercially available column-based RNA preparation kit. BioTechniques 40:54-58.

2. Buzelle, S.L., R.E. MacPherson, W.T. Peppler, L. Castellani, and D.C. Wright. 2015. The contribution of IL-6 to beta 3 adrenergic receptor mediated adipose tissue remodeling. Physiol. Rep. 3:e12312.

3. Simões, A.E., D.M. Pereira, J.D. Amaral, A.F. Nunes, S.E. Gomes, P.M. Rodrigues, A.C. Lo, R. D'Hooge, et al. 2013. Efficient recovery of proteins from multiple source samples after TRIzol(®) or TRIzol(@)LSRNA extraction and long-term storage. BMC Genomics 14:181.

4. Nestler, E.J. and P. Greengard. 1999. Protein phosphorylation is of fundamental importance in biological regulation. In G.J. Siegel, B.W. Granoff, and R.W. Albers, et al., (Eds.), Basic Neurochemistry: Molecular, Cellular and Medical Aspects, 6th edition. Lippincott-Raven, Philadelphia, PA.

5. Humphrey, S.J., D.E. James, and M. Mann. 2015. Protein Phosphorylation: A Major Switch Mechanism for Metabolic Regulation. Trends Endocrinol. Metab. 26:676-687.

Ousseynou Sarr, David J. Dyck, and David M. Mutch

Department of Human Health and Nutritional Sciences, University of Guelph, Guelph, ON, Canada

BioTechniques 61:233-235 (November 2016) doi 10.2144/000114471

Keywords: protein isolation; phosphoproteins

Supplementary material for this article is available at www.BioTechniques.com/114471.

Received 24 April 2016; accepted 04 August 2016.

Address correspondence to David M. Mutch, Department of Human Health and Nutritional Sciences, University of Guelph, Guelph, ON N1G 2W1 Canada. E-mail: dmutch@uoguelph.ca

To purchase reprints of this article, contact: biotechniques@fosterprinting.com analytikjena

Small Animal In Vivo Imaging System

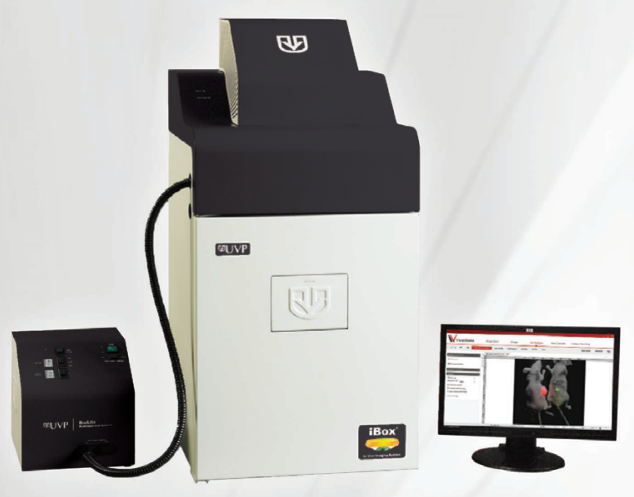

\section{UVP iBox ${ }^{\circledR}$ Scientia ${ }^{T M}$}

Bioluminescence and fluorescence applications

- Cancer research

- Small animal imaging

- In vivo

Tumor studies

Heart disease

Gene expression

Inflammation

Metastasis

Bacterial/viral infections

Immunology

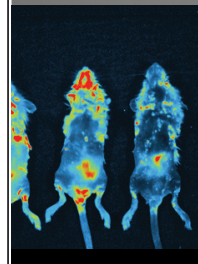

बUVP

An Analytik Jena Company

www.uvp.com

UVP, LLC USA (800) 452-6788

Cambridge UK +44(0) 1223-420022 